\title{
Diacronie
}

Studi di Storia Contemporanea

$\mathrm{N}^{\circ} 29,1 \mid 2017$

"Crash test"

\section{Maurizio Isabella, Konstantina Zanou (edited by), Mediterranean Diasporas. Politics and Ideas in the Long 19th Century}

Deborah Paci

\author{
(2) OpenEdition \\ Journals \\ Edizione digitale \\ URL: http://journals.openedition.org/diacronie/5137 \\ DOI: 10.4000/diacronie.5137 \\ ISSN: 2038-0925 \\ Editore \\ Association culturelle Diacronie
}

Notizia bibliografica digitale

Deborah Paci, « Maurizio Isabella, Konstantina Zanou (edited by), Mediterranean Diasporas. Politics and Ideas in the Long 19th Century », Diacronie [Online], № 29, 1 | 2017, documento 19, Messo online il 29 mars 2017, consultato il 24 septembre 2020. URL : http://journals.openedition.org/diacronie/5137 ; DOI : https://doi.org/10.4000/diacronie.5137 


\section{Diacronie}

Studi di Storia Contemporanea

29, 1/2017

"Crash test". Continuità, discontinuità, legami e rotture nelle dinamiche della storia contemporanea

\section{RECENSIONE: Maurizio ISABELLA, Konstantina ZANOU (edited by), Mediterranean Diasporas. Politics and Ideas in the Long 19th Century, London-New Delhi-New York-Sydney, Bloomsbury, 2016, 217 pp.}

A cura di Deborah PACI

Per citare questo articolo:

PACI, Deborah, «RECENSIONE: Maurizio ISABELLA, Konstantina ZANOU (edited by), Mediterranean Diasporas. Politics and Ideas in the Long 19th Century, London-New Delhi-New York-Sydney, Bloomsbury, 2016, 217 pp.», Diacronie. Studi di Storia Contemporanea : "Crash test". Continuità, discontinuità, legami e rotture nelle dinamiche della storia contemporanea, 29, 1/2017, 29/3/2017,

URL: < http://www.studistorici.com/2017/3/29/paci_numero_29/ >

Diacronie Studi di Storia Contemporanea $\rightarrow$ http://www.diacronie.it Rivista storica online. Uscita trimestrale.

redazione.diacronie@hotmail.it

Comitato di direzione: Naor Ben-Yehoyada - João Fábio Bertonha - Christopher Denis-Delacour - Maximiliano Fuentes Codera Anders Granås Kjøstvedt - John Paul Newman - Deborah Paci - Niccolò Pianciola - Spyridon Ploumidis - Wilko Graf Von Hardenberg

Comitato di redazione: Jacopo Bassi - Luca Bufarale - Gianluca Canè - Fausto Pietrancosta - Alessandro Salvador - Matteo Tomasoni Diritti: gli articoli di Diacronie. Studi di Storia Contemporanea sono pubblicati sotto licenza Creative Commons 3.0. Possono essere riprodotti e modificati a patto di indicare eventuali modifiche dei contenuti, di riconoscere la paternità dell'opera e di condividerla allo stesso modo. La citazione di estratti è comunque sempre autorizzata, nei limiti previsti dalla legge. 


\section{9/ RECENSIONE: Maurizio ISABELLA, Konstantina ZANOU (edited by), Mediterranean Diasporas. Politics and Ideas in the Long 19th Century, London-New Delhi-New York-Sydney, Bloomsbury, 2016, 217 pp.}

È possibile tracciare una storia transnazionale del Mediterraneo durante il XIX secolo? È possibile farlo studiando profili biografici di intellettuali che agirono a tutti gli effetti come attori transnazionali?

Un tentativo di risposta a questi interrogativi è contenuta in Mediterranean Diasporas. Politics and Ideas in the Long 19th Century ${ }^{1}$.

La varietà e la ricchezza della produzione intellettuale - soprattutto durante il XIX secolo sono al centro della raccolta di saggi curata da Maurizio Isabella ${ }^{2}$ e Konstantina Zanou ${ }^{3}$. Il punto di vista dei curatori - enucleato nell'introduzione del volume - è che non esista una specificità mediterranea, e che lo sforzo degli studiosi debba orientarsi nella direzione di ricollocare le convergenze e le corrispondenze intellettuali e culturali tra le sponde del Mediterraneo in una prospettiva più ampia, evitando discorsi «mediterraneisti»4.

L'obiettivo di questi saggi è «[...] rethink the boundaries of their various disciplines [...]»; in questo modo il corpus dei testi

\footnotetext{
${ }^{1}$ ISABELLA, Maurizio, ZANOU, Konstantina (edited by), Mediterranean Diasporas. Politics and Ideas in the Long 19th Century, London - New Delhi - New York - Sydney, Bloomsbury, 2016.

${ }^{2}$ Maurizio Isabella è Senior lecturer in Modern History alla Queen Mary University di Londra. Ha pubblicato Risorgimento in Exile. Italian Émigrés and the Liberal International in the Post-Napoleonic Era, Oxford, Oxford University Press, 2009, che è stato in italiano con il titolo Risorgimento in Esilio, Roma-Bari, Laterza, 2011, oltre ad avere pubblicato un grande numero di articoli e saggi in opere miscellanee sul Risorgimento.

3 Konstantina Zanou è Assistant professor presso la Columbia University di New York e résident dell'IEA (Institut d'Études Avancées) di Parigi. Nel 2017 uscirà la sua monografia Transnational Patriotism in the Mediterranean 1800-1830: Stammering the Nation, Oxford, Oxford University Press. Si è occupata dei milieux intellettuali greci e italiani nel XIX secolo.

4 Ibidem, p. 16.
} 
[...] explore new and often neglected connections, parallels and similarities that suggest a revision both of the way in which the Mediterranean is perceived, and the manner in which political imagination and ideas were shaken in different areas 5 .

Lo spazio marittimo mediterraneo nel XIX secolo è quello in cui aumenta la distanza fra Oriente e Occidente, fra Nord e Sud anche per effetto di una polarizzazione culturale ${ }^{6}$. La nozione di arretratezza è infatti stata impiegata a più riprese - durante questo periodo - per giustificare il colonialismo.

Il Mediterraneo viene tuttavia re-immaginato come spazio geografico e di civilizzazione: del resto il Diciannovesimo secolo è quello in cui l'apertura del canale di Suez e la realizzazione di una fitta rete ferroviaria sulle coste mediterranee contribuiscono a rilanciarne l'importanza e il ruolo nel mondo. Il Mediterraneo ritrova una centralità anche attraverso l'uso del passato che viene operato dagli intellettuali: la giustificazione di nuove mire espansionistiche e l'elogio della colonizzazione delle popolazioni pre-romane sono temi che riecheggiano nel lavoro degli intellettuali dell'area in quest'epoca7.

Il libro si articola in dieci capitoli, ciascuno dedicato a specifici aspetti della storia delle idee nel Mediterraneo nel XIX secolo.

Il primo, Letters from Spain: The 1820 Revolution and the Liberal International ${ }^{8}$, di Juan Luis Simal, affronta il tema della filiazione della rivoluzione spagnola del 1820. Simal nel paragrafo introduttivo si sofferma su una breve introduzione al genere epistolare e ai suoi utilizzi nel XVIII e XIX secolo per poi affrontare più specificamente l'impatto delle vicende che videro protagonista la Penisola iberica nel primo quarto dell'Ottocento. Il saggio mette in luce la fitta rete di relazioni e di contatti intessuti sia sulle sponde del Mediterraneo, sia oltreoceano, dando avvio a un dibattito paneuropeo in campo liberale (e a un corrispettivo interscambio) intellettuale transcontinentale.

Gabriel Paquette $^{9}$ ripercorre invece la vicenda umana di Almeida Garrett, uno dei più importanti letterati e politici dell'Ottocento portoghese. La rivoluzione del 1820 in Portogallo rappresentò l'occasione per il consolidamento di un legame intellettuale fra i liberali iberici e quelli d'Oltreoceano. È in questo e nel successivo contesto che agì Almeida Garrett: l'intento del saggio è quello di tracciarne un percorso politico tra il 1820 e il 1834, anni durante i quali partecipò alla vita politica sia in patria che in esilio.

\footnotetext{
5 Ibidem, p. 10.

6 Ibidem, p. 9.

7 Ibidem, p. 10.

8 SIMAL, Juan Luis, Letters from Spain: The 1820 Revolution and the Liberal International, in ISABELLA, Maurizio, ZANOU, Konstantina, op. cit., pp. 25-41.

9 PAQUETTE, Gabriel, An Itinerant Liberal: Almeida Garrett's Exilic Itineraries and Political Ideas in the Age of Southern European Revolutions (1820-34), in ISABELLA, Maurizio, ZANOU, Konstantina, op. cit., pp. 43-57.
} 
Paquette si sofferma in particolare sul concetto di liberdade meridional, sviluppato soprattutto in Portugal na Balança da Europa ${ }^{10}$; Garrett aveva infatti operato una distinzione fra paesi del Sud e del Nord dell'Europa, anche sul piano politico. La libertà era da intendersi in due differenti accezioni: da una parte la libertà sul piano politico interno (ad esempio un governo costituzionale) e dall'altra quella fra nazioni (la sovranità nazionale). Il Portogallo avrebbe dovuto ambire a tornare a quel sistema di libertà conseguito nel Medioevo, prima che la colonizzazione oltremare cancellasse la libertà dai paesi della penisola iberica, accentrando il potere nelle mani di governi dispotici.

Learning Lessons from the Iberian Peninsula ${ }^{11}$ di Grégoire Bron esamina l'impatto delle rivoluzioni che ebbero luogo nella penisola iberica sulla cultura politica di ben due generazioni di italiani. Un'attenta analisi delle società spagnola e portoghese - sostiene Bron - portò gli esuli italiani a rivedere e riconsiderare le basi del loro impegno politico rivoluzionario in Italia. Le loro esperienze nella penisola iberica li indussero a considerare con pessimismo la possibilità di realizzare istituzioni politiche moderne sulla base della partecipazione popolare.

Mediterranean Liberals? Italian Revolutionaries and the Making of a Colonial Sea, c.a. 1800-183012, tratta degli itinerari degli intellettuali liberali italiani nella prima metà dell'Ottocento, nel contesto della graduale trasformazione del Mediterraneo in un "lago britannico". La situazione geopolitica (e culturale) vide queste figure muoversi in uno spazio mediterraneo contrassegnato dalla presenza di due imperi: quello britannico e quello francese. Come dimostra Isabella, spostandosi tra le periferie (insulari e non) e i centri - rappresentati dalle due capitali, Londra e Parigi - i liberali italiani entrarono in contatto con nuove idee e con altri intellettuali e politici.

Il saggio di Ian Coller, Ottomans on the $\mathrm{Move}^{13}$, si sofferma invece sul contesto della circolazione di idee tra gli intellettuali ottomani e in particolare su una figura: quella di Hassuna D'Ghies. Originario di Tripoli, divenne direttore de «Le Moniteur Ottoman» nel gennaio 1836 e ricoprì questa carica solamente per pochi mesi, durante i quali si batté per la modernizzazione dell'Impero ottomano: una modernizzazione che potesse opporsi al dominio dell'imperialismo europeo e non divenirne un'appendice. L'intento di Coller è quello di mostrare come i viaggi e i soggiorni di D'Ghies a Tripoli, Parigi, Marsiglia, Londra e Istanbul ne influenzarono il pensiero.

\footnotetext{
10 GARRETT, Almeida, Portugal na balança da Europa: do que tem sido e do que ora lhe convém ser na nova ordem de coisas do mundo civilizado, London, Sustenance, 1830.

${ }^{11}$ BRON, Grégoire, Learning Lessons from the Iberian Peninsula: Italian Exiles and the Making of a Risorgimento Without People, 1820-48, in ISABELLA, Maurizio, ZANOU, Konstantina, op. cit., pp. 59-76.

12 ISABELLA, Maurizio, Mediterranean Liberals? Italian Revolutionaries and the Making of a Colonial Sea, c.a. 18001830, in ISABELLA, Maurizio, ZANOU, Konstantina, op. cit., pp. 77-96.

13 COLLER, Ian, Ottomans on the Move: Hassuna D'Ghies and the 'New Ottomanism' of the 1830s, in ISABELLA, Maurizio, ZANOU, Konstantina, op. cit., pp. 97-115.
} 
Konstantina Zanou nel suo Imperial Nationalism and Orthodox Enlightenment: a Diasporic Story Between the Ionian Islands, Russia and Greece, c.a. 1800-3014 si sofferma sull'impatto prodotto dalla presenza russa sulle coste del Mediterraneo - e in particolare nelle isole Ionie - nello sviluppo delle idee liberali. Zanou dimostra come la visione - storiograficamente consolidata ${ }^{15}$ - che vede l'illuminismo greco come una filiazione della rivoluzione francese e un'eredità illuminista, rappresenti, in realtà, solo una lettura parziale del fenomeno. È così sorta una corrente storiografica che, a partire dalla premessa che non sia mai esistita un'opposizione tout court fra cristianesimo e illuminismo, ha affermato che si può parlare di "illuminismo neoellenico" come di un fenomeno con caratteristiche peculiari. Focalizzandosi sul caso delle isole Ionie, Zanou mette in evidenza - prendendo in esame il pensiero di alcuni intellettuali - come l'idea di nazione moderna non fosse incompatibile con il mondo cristiano.

Il saggio di Dominique Kirchner Reill Away or Homeward Bound? The slippery Case of Mediterranean Place in the Era Before Nation-states ${ }^{16}$, parte dall'intento di dimostrare una tesi: i luoghi del Mediterraneo dell'epoca precedente all'avvento degli Stati-Nazione non possono essere trattati come categorie stabili. Per dimostrare questo, l'autrice ricorre a un'analisi comparata fra tre intellettuali attivi durante - o immediatamente a seguito de - le guerre napoleoniche: Niccolò Tommaseo, Pacifico Valussi e Matija Ban.

The Strange Lives of Ottoman Liberalism ${ }^{17}$ di Andrew Arsan si occupa degli orientamenti politici di Mustafa Fatil Paşa, sostenitore politico ed economico dei Giovani Ottomani. Analizzando il suo epistolario, l'autore riscontra una consonanza di temi con quelli allora discussi dai liberali europei, dimostrando così - oltre ad una capillare circolazione delle idee - una medesima preoccupazione in seno agli intellettuali dell'epoca.

Il saggio di Artan Puto e Maurizio Isabella ${ }^{18}$ è invece consacrato alla nascita e all'evoluzione dell'idea politica e culturale di nazione albanese. Come viene sottolineato dai due autori nel paragrafo introduttivo al capitolo, il Nation-building albanese necessitò - più ancora che in altri casi - che venisse elaborata ex novo un'idea di nazione abbastanza forte da poter rendere coese popolazioni differenti per lingua e religione. Puto e Isabella esaminano questo processo prendendo in esame due fra le più eminenti figure di intellettuali albanesi: Girolamo De Rada e

\footnotetext{
14 ZANOU, Konstantina, Imperial Nationalism and Orthodox Enlightenment: a Diasporic Story Between the Ionian Islands, Russia and Greece, c.a. 1800-30, in ISABELLA, Maurizio, ZANOU, Konstantina, op. cit., pp. 117-134.

15 Si veda, ad esempio, la produzione di Paschalis Kitromilides. Tra gli altri: KITROMILIDES, Paschalis M., Enlightenment and Revolution. The Making of Modern Greece, Cambridge (Mass.), Harvard University Press, 2013. ${ }^{16}$ KIRCHNER REILL, Dominique, Away or Homeward Bound? The slippery Case of Mediterranean Place in the Era Before Nation-states, in ISABELLA, Maurizio, ZANOU, Konstantina, op. cit., pp. 135-152.

17 ARSAN, Andrew, The Strange Lives of Ottoman Liberalism: Exile, Patriotism and Constitutionalism in the Thought of Mustafa Fazil Paşa, in ISABELLA, Maurizio, ZANOU, Konstantina, op. cit., pp. 153-170.

18 PUTO, Artan, ISABELLA, Maurizio, From Southern Italy to Istanbul: Trajectories of Albanian Nationalism in the Writings of Girolamo de Rada and Shemseddin Sami Frashëri, ca. 1848-1903, in ISABELLA, Maurizio, ZANOU, Konstantina, op. cit., pp. 171-187.
} 
Sami Frashëri. La visione della nazione albanese, come del resto i profili biografici di questi due intellettuali, era differente: ad unirli fu il fatto che entrambi cercassero di giungere ad una definizione della cultura albanese a partire dalla loro personale interazione con differenti tradizioni culturali e intellettuali. Entrambe le soluzioni della "questione albanese" a cui giunsero, contemplavano la permanenza del mondo multiculturale che caratterizzava l'Impero ottomano.

Vangelis Kechriotis in Ottomanism with a Greek Face ${ }^{19}$ prende in esame la diaspora dei greci di Cappadocia e il lealismo di alcuni greci nei confronti dell'Impero ottomano. Dopo aver introdotto il tema, offrendo al lettore qualche suggestione cinematografica e uno stato dell'arte su questo tema, l'autore si sofferma sui profili biografici di due greci di Cappadocia successivamente emigrati a Smyrne: Pavlos Carolidis ed Emmanouil Emmanouilidis. Entrambi divennero importanti membri della comunità smirniota e rimasero fedeli all'Impero ottomano rifiutando di schierarsi con i greci "irredentisti" che auspicavano l'inclusione delle terre da loro abitate nello Stato greco, almeno finché la dinamica dei nazionalismi non li costrinse a rivedere la loro posizione.

Chiude il volume una postfazione di Thomas Gallant ${ }^{20}$ che inquadra la raccolta miscellanea nel campo emergente degli studi transnazionali. I saggi di questo volume sono infatti connotati da un comune approccio che si sta facendo largo in quest'ambito:

[...] an approach that has emerged as one of the most important in transnational history is biography. A number of scholars have shown the analytical utility of tracing careers and movement of individuals, families and groups, such as financiers, merchants or soldiers, so as to uncover broader historical process like imperialism or the spread of capitalism ${ }^{21}$.

I saggi di Mediterranean Diasporas contribuiscono allo studio del cosmopolitismo intellettuale, rimettono in discussione il concetto di perifericità del Mediterraneo nel XIX secolo e, soprattutto, l'idea dello studio biografico a partire dal concetto di nazione. Un concetto del resto ribadito chiaramente da Dominique Kirchner Reill in uno dei saggi quando afferma che «[...] 'place', in the Mediterranean before Nation-States should not be treated as a stable category, but instead should be read against the modern geographical grain, especially when analysing the mental world of the Mediterranean's provincial imperial subjects» ${ }^{22}$.

\footnotetext{
19 KECHRIOTIS, Vangelis, Ottomanism with a Greek Face: Karamanli Greek Orthodox Diaspora at the End of the Ottoman Empire, in ISABELLA, Maurizio, ZANOU, Konstantina, op. cit., pp. 189-204.

20 GALLANT, Thomas, Afterword: Writing Mediterranean Diasporas After the Transnational Turn, in ISABELLA, Maurizio, ZANOU, Konstantina, op. cit., pp. 205-209.

${ }^{21}$ Ibidem, p. 206.

22 Ibidem, p. 136.
} 
Il senso e l'importanza di questo lavoro collettaneo sono sinteticamente espressi, ancora una volta, da Gallant nella postfazione:

[...] it will be hard to think about Nineteenth-century liberalism, nationalism and the revolutions that they spawned without factoring in the key role played by diasporic mediterranean intellectuals. Not only did these transnational and cosmopolitan refugees and migrants contribute significantly to modern state and nation-building in the Mediterranean but they were vitally important actors in movements that helped to shape the very foundations of the modern world 23 .

23 Ibidem, p. 209. 


\section{L'AUTORE}

Deborah PACI è assegnista di ricerca presso il Dipartimento di Studi linguistici e culturali comparati dell'Università Ca' Foscari di Venezia e ricercatrice associata al Centre de la Méditerranée Moderne et Contemporaine dell'Université de Nice Sophia Antipolis. È autrice delle monografie: L'arcipelago della pace. Le isole Åland e il Baltico (XIX-XXI sec.), Milano, Unicopli, 2016; Corsica fatal, Malta baluardo di romanità. L'irredentismo fascista nel Mare nostrum (1922-1942), Firenze, Le Monnier-Mondadori Education, 2015.

URL: < http://www.studistorici.com/2009/02/24/deborah-paci/ > 